Supplementary Information for:

\title{
Size and Quality Enhancement of 2D Semiconducting Metal-Organic Chalcogenolates by Amine Addition
}

Watcharaphol Paritmongkol ${ }^{1,2}$, Tomoaki Sakurada ${ }^{2}$, Woo Seok Lee ${ }^{2,3}$, Ruomeng Wan ${ }^{1,2}$, Peter Müller ${ }^{1}$, William A. Tisdale ${ }^{2 *}$

${ }^{1}$ Department of Chemistry, Massachusetts Institute of Technology, Cambridge, Massachusetts 02139, United States

${ }^{2}$ Department of Chemical Engineering, Massachusetts Institute of Technology, Cambridge, Massachusetts 02139, United States

${ }^{3}$ Department of Materials Science and Engineering, Massachusetts Institute of Technology, Cambridge, Massachusetts 02139, United States

*Correspondence to: tisdale@mit.edu

\section{Contents}

1. Supplementary Figures $\quad$ S2

2. Additional Crystallographic Information of AgSePh and AgSePhMe S7 


\section{Supplementary Figures}

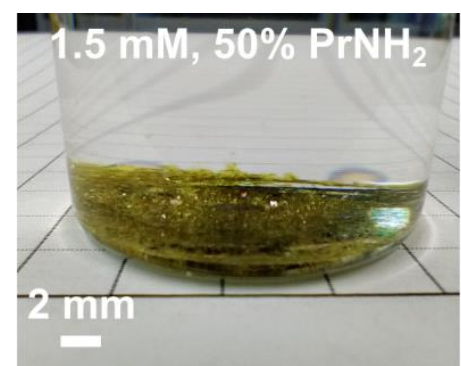

Figure S1. AgSePh crystals formed by a single-phase reaction after mixing $10 \mathrm{~mL}$ of $3 \mathrm{mM}$ solution of $\mathrm{AgNO}_{3}$ in $\mathrm{PrNH}_{2}$ with $10 \mathrm{~mL}$ of $3 \mathrm{mM}$ solution of $\mathrm{Ph}_{2} \mathrm{Se}_{2}$ in toluene for 5 days at room temperature.

(a)

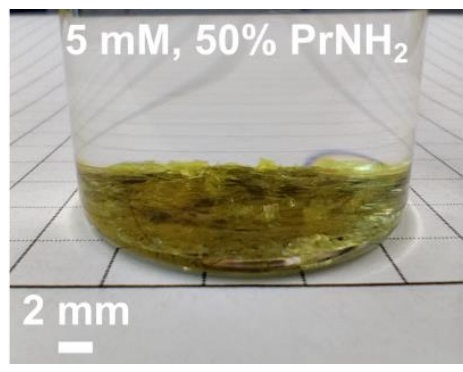

(b)

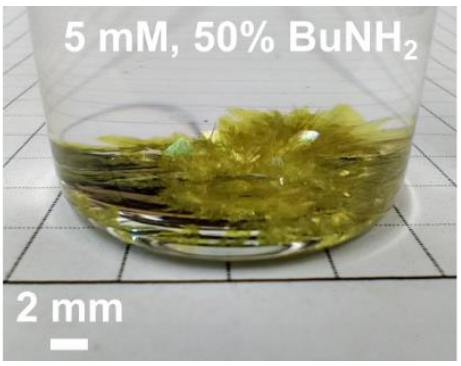

(c)

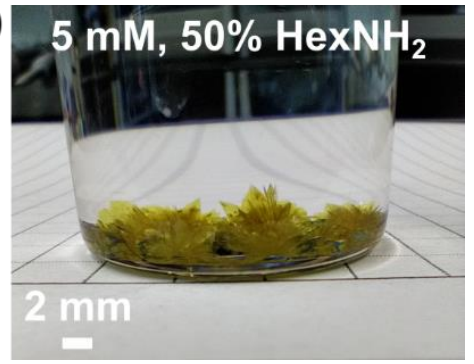

Figure S2. AgSePh crystals formed by single-phase reactions after storing a $5 \mathrm{mM}$ mixture of $\mathrm{AgNO}_{3}$ and $\mathrm{Ph}_{2} \mathrm{Se}_{2}$ in $50 \% \mathrm{v} / \mathrm{v}$ (a) $\mathrm{PrNH}_{2}$ /toluene, (b) $\mathrm{BuNH}_{2} /$ toluene, and (c) $\mathrm{HexNH}_{2} /$ toluene for 5 days at room temperature.

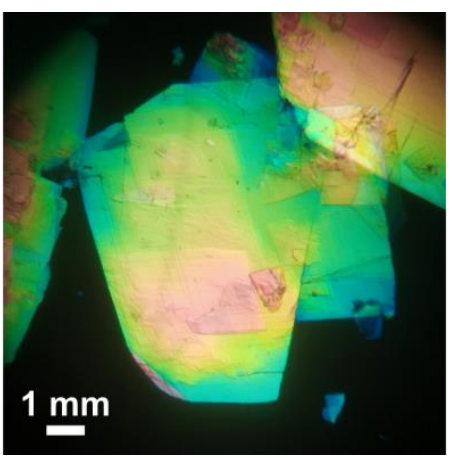

Figure S3. Polarized optical micrograph of representative crystals formed after storing a $10 \mathrm{mM}$ mixture of $\mathrm{AgNO}_{3}$ and $\mathrm{Ph}_{2} \mathrm{Se}_{2}$ in $50 \% \mathrm{v} / \mathrm{v} \mathrm{HexNH} \mathrm{H}_{2}$ /toluene for 5 days at room temperature. 


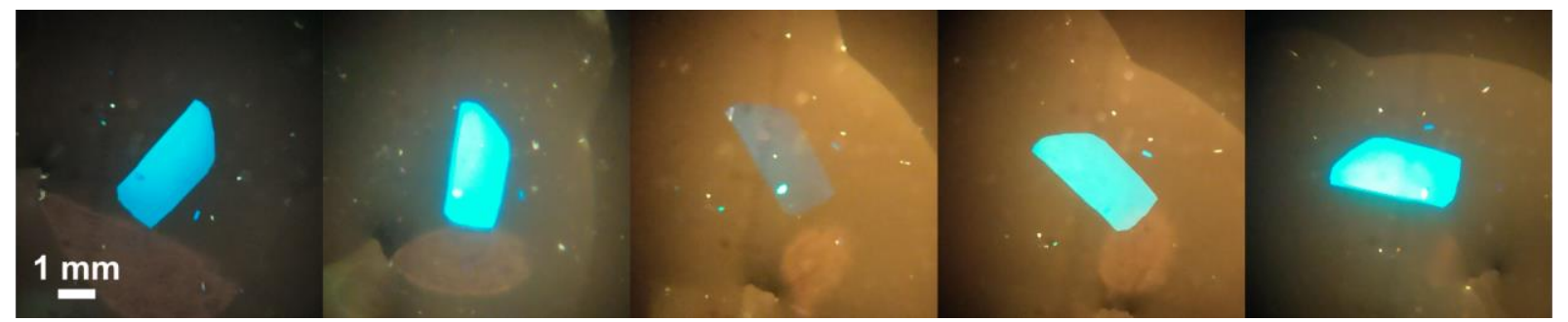

Figure S4. Polarized optical micrographs of an isolated crystal rotated at different angles with respect to light polarization. The uniform brightness throughout the crystal at each rotation angle suggests that the crystal is single.

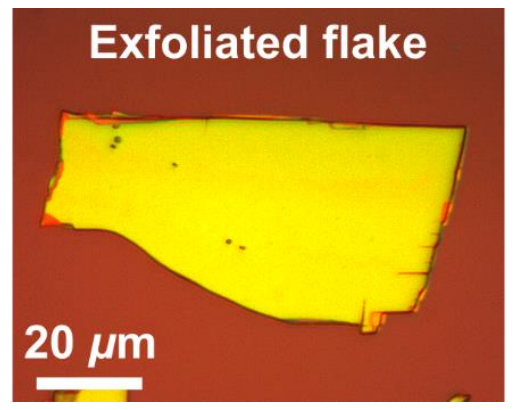

Figure S5. Optical micrograph of an exfoliated flake by tape. 

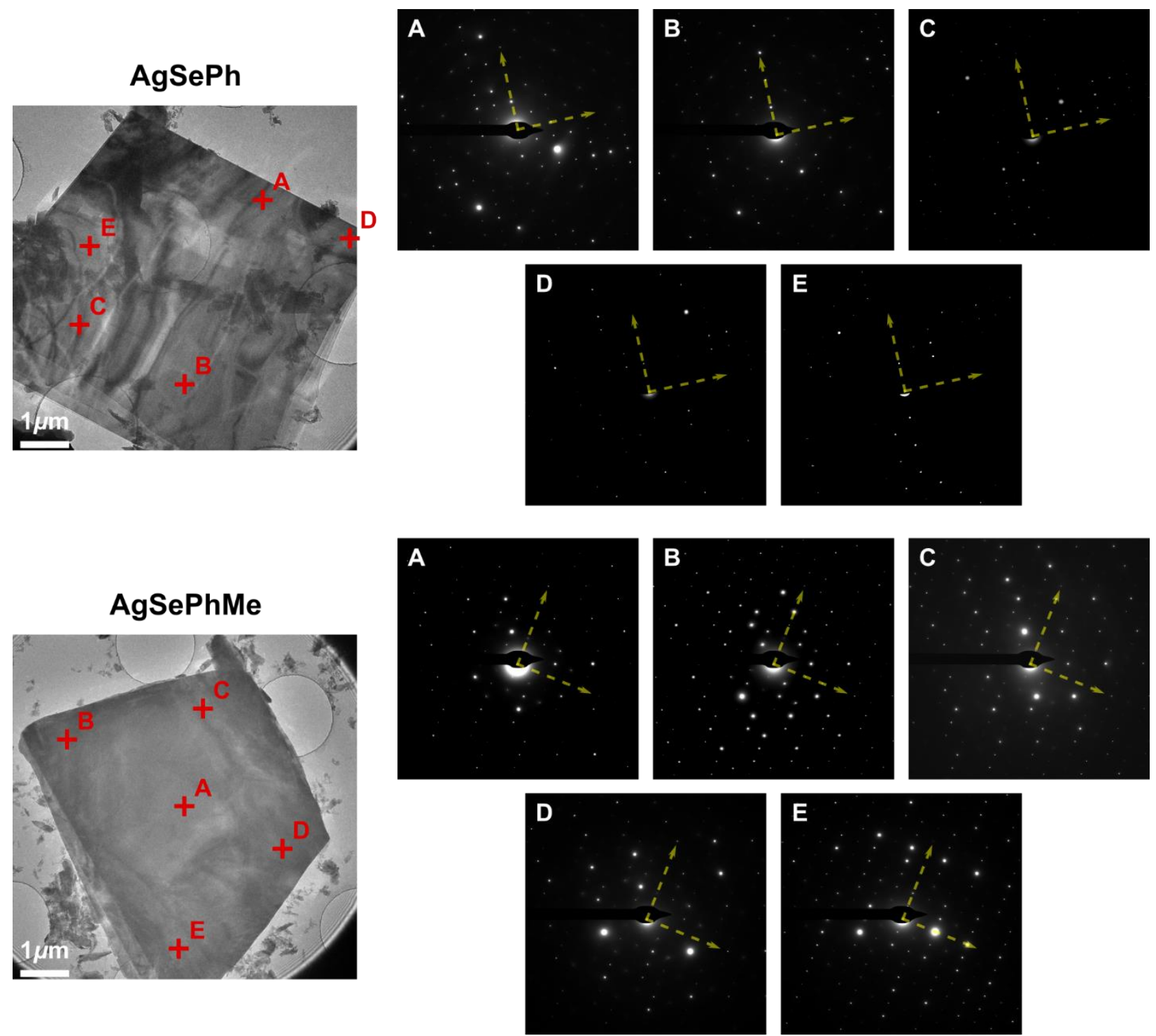

Figure S6. TEM images of exfoliated $\mathrm{AgSePh}$ and $\mathrm{AgSePhMe}$ crystals along with electron diffraction at marked locations. The electron diffraction data at different locations throughout the exfoliated crystals show the same diffraction orientation, suggesting the exfoliated crystals are single and do not contain large twin domains. 

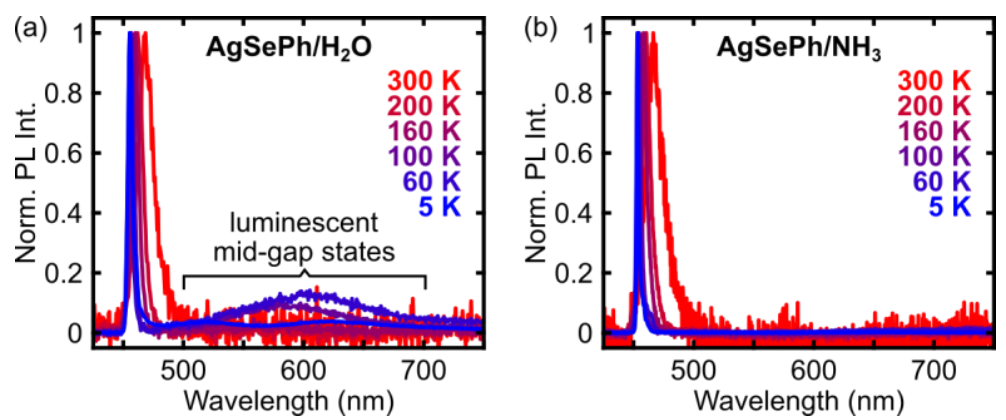

Figure S7. Selected temperature-dependent photoluminescence spectra between $5 \mathrm{~K}$ and $300 \mathrm{~K}$ of AgSePh crystals prepared in (a) $\mathrm{H}_{2} \mathrm{O}$ and in (b) $\mathrm{NH}_{3} / \mathrm{H}_{2} \mathrm{O}$.

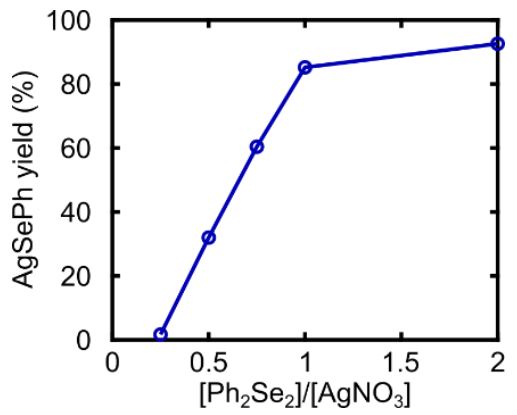

Figure S8. Determination of reaction stoichiometry of $\mathrm{Ph}_{2} \mathrm{Se}_{2}$ and $\mathrm{AgNO}_{3}$. 

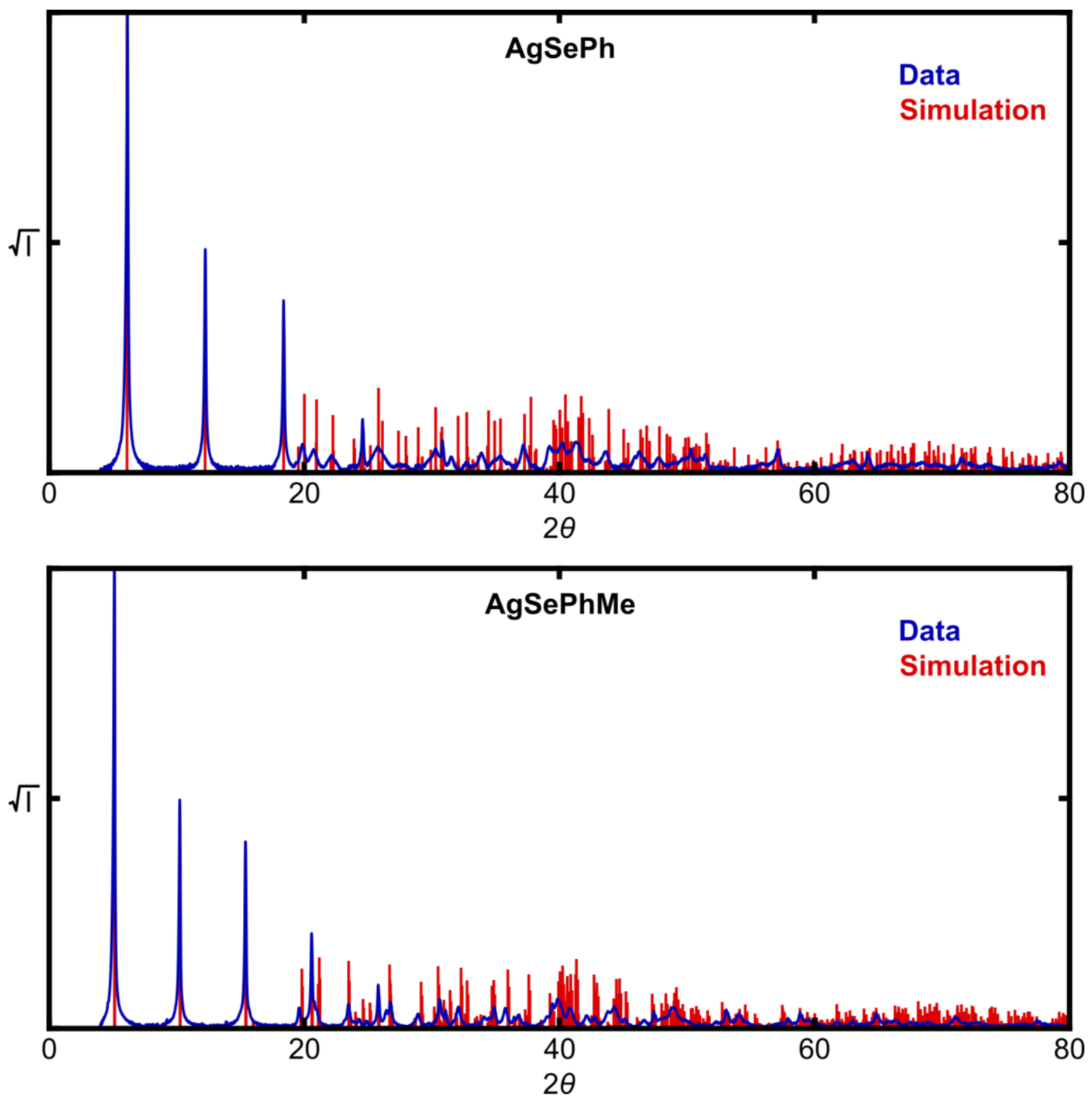

Figure S9. Powder X-ray diffraction patterns (blue) of AgSePh (top) and AgSePhMe (bottom) and the corresponding simulated patterns (red) from collected crystallographic information by single-crystal X-ray diffraction. 


\section{Additional Crystallographic Information of AgSePh and AgSePhMe}

The two crystal structures are similar (Figure S10). Both compounds crystallize in the monoclinic centrosymmetric space group $P 2_{1} / c$ : $\mathrm{AgSePh}$ with two monomeric subunits in the asymmetric unit (contents of asymmetric unit: $\mathrm{Ag}_{2} \mathrm{Se}_{2} \mathrm{Ar}_{2} ; \mathrm{Ar}=\mathrm{Ph}=$ phenyl or $\mathrm{C}_{6} \mathrm{H}_{5}$ ) and $\mathrm{AgSePhMe}$ with one monomeric subunit in the asymmetric unit (contents of asymmetric unit: AgSeAr; Ar = 4-methyl-phenyl or $\mathrm{C}_{7} \mathrm{H}_{7}$ ). Both structures are 2D-polymers and application of crystallographic symmetry gives rise to infinite sheets with the phenyl rings displayed on both surfaces. Those sheets stack along the crystallographic $c$-direction for the structure of AgSePh and along the crystallographic $a$-direction for the structure of $\mathrm{AgSePhMe}$ in such a fashion that the phenyl rings from neighboring sheets meet in a hydrophobic layer that, in the crystallographic packing, alternates with a metallic layer of selenium and silver.
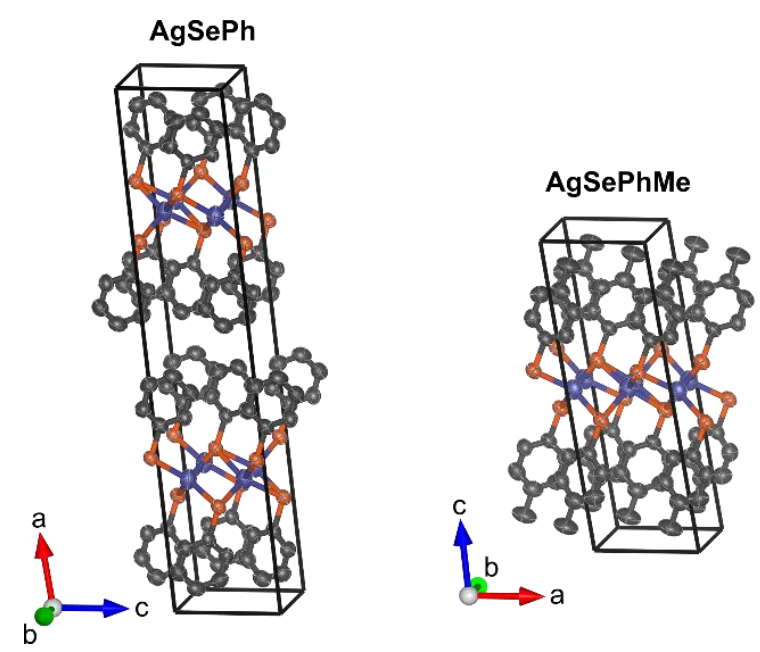

Figure S10. Crystallographic unit cells of $\mathrm{AgSePh}$ and $\mathrm{AgSePhMe}$. Thermal ellipsoids of $\mathrm{Ag}$, $\mathrm{Se}$, and $\mathrm{C}$ are depicted in purple, orange, and black, respectively. $\mathrm{H}$ atoms are omitted for clarity.

The crystal quality of $\mathrm{AgSePh}$ was comparatively low and there were signs of nonmerohedral twinning. The twinning could not be resolved. It seems that in addition to one large, dominating major single-crystal domain, there are several minor domains that, individually, are too small to allow for stable data reduction, but together, they comprise an appreciable portion of the crystal so that some effects of the twinning are apparent in the structure. Most notably, there is unexplained positive and negative residual electron density located at seemingly random sites in the structure, which may well be an artefact of unresolved non-merohedral twinning. The highest residual density maximum corresponds to 1.82 electrons and is located $1.65 \AA$ away from selenium 
atom Se2; the lowest negative peak corresponds to -2.42 electrons and is located $1.66 \AA$ away from selenium atom Se1. The two phenyl rings were restrained to display similar geometry with respect to bonds and angles. No disorder was included in the model to refine the phenyl rings in the structure. The minor crystal domains observed were mainly caused by new crystal nucleation when the reaction solution was removed from the freezer at $-20{ }^{\circ} \mathrm{C}$ to the ambient temperature during the SCXRD sample preparation step.

The structure of $\mathrm{AgSePhMe}$ was refined as a pseudo-merohedral twin (twin law -1 0 -1 0 -1000 1). The twin ratio was refined freely and converged at 0.0258(3). In spite of the small size of the minor twin domain, introduction of the twin law improved the structure markedly. No restraints were applied. No disorder was included in the model to refine the phenyl rings in the structure. 
Table S1. Atomic coordinates (x 104) and equivalent isotropic displacement parameters $\left(\AA^{2} \mathrm{x}\right.$ $\left.10^{3}\right)$ for $\mathrm{AgSePh} . \mathrm{U}(\mathrm{eq})$ is defined as one third of the trace of the orthogonalized $\mathrm{Uij}^{\mathrm{ij}}$ tensor.

\begin{tabular}{lrrrr}
\hline & $\mathrm{x}$ & $\mathrm{y}$ & $\mathrm{z}$ & $\mathrm{U}(\mathrm{eq})$ \\
\hline $\mathrm{Ag}(1)$ & $2373(1)$ & $11284(1)$ & $2488(1)$ & $18(1)$ \\
$\mathrm{Se}(1)$ & $-469(1)$ & $9292(1)$ & $2964(1)$ & $13(1)$ \\
$\mathrm{C}(1)$ & $5866(5)$ & $4350(4)$ & $3581(1)$ & $14(1)$ \\
$\mathrm{C}(2)$ & $4574(5)$ & $5121(5)$ & $3912(1)$ & $17(1)$ \\
$\mathrm{C}(3)$ & $5447(6)$ & $5105(5)$ & $4376(1)$ & $20(1)$ \\
$\mathrm{C}(4)$ & $7583(6)$ & $4323(5)$ & $4507(1)$ & $19(1)$ \\
$\mathrm{C}(5)$ & $8875(6)$ & $3571(5)$ & $4175(1)$ & $20(1)$ \\
$\mathrm{C}(6)$ & $8027(5)$ & $3596(4)$ & $3710(1)$ & $17(1)$ \\
$\mathrm{Ag}(2)$ & $2594(1)$ & $7313(1)$ & $2540(1)$ & $18(1)$ \\
$\mathrm{Se}(2)$ & $4572(1)$ & $4302(1)$ & $2947(1)$ & $13(1)$ \\
$\mathrm{C}(11)$ & $810(5)$ & $9284(4)$ & $3602(1)$ & $14(1)$ \\
$\mathrm{C}(12)$ & $-514(5)$ & $8585(5)$ & $3933(1)$ & $17(1)$ \\
$\mathrm{C}(13)$ & $322(6)$ & $8655(5)$ & $4399(1)$ & $20(1)$ \\
$\mathrm{C}(14)$ & $2466(6)$ & $9431(5)$ & $4532(1)$ & $20(1)$ \\
$\mathrm{C}(15)$ & $3794(5)$ & $10119(5)$ & $4196(1)$ & $19(1)$ \\
$\mathrm{C}(16)$ & $2972(5)$ & $10041(4)$ & $3731(1)$ & $17(1)$ \\
& & & & \\
\hline & & & &
\end{tabular}


Table S2. Bond lengths $[\AA]$ and angles $\left[{ }^{\circ}\right]$ for $\mathrm{AgSePh}$.

\begin{tabular}{|c|c|c|c|}
\hline $\operatorname{Ag}(1)-\operatorname{Se}(1)$ & $2.6878(4)$ & $\operatorname{Se}(1) \# 2-\operatorname{Ag}(1)-\operatorname{Ag}(2)$ & $146.986(14)$ \\
\hline $\operatorname{Ag}(1)-\operatorname{Se}(2) \# 1$ & $2.7013(4)$ & $\operatorname{Se}(2) \# 3-\operatorname{Ag}(1)-\operatorname{Ag}(2)$ & $137.623(13)$ \\
\hline $\operatorname{Ag}(1)-\operatorname{Se}(1) \# 2$ & $2.7353(4)$ & $\operatorname{Se}(1)-\operatorname{Ag}(1)-\operatorname{Ag}(2) \# 2$ & $60.250(10)$ \\
\hline $\operatorname{Ag}(1)-\operatorname{Se}(2) \# 3$ & $2.8155(4)$ & $\operatorname{Se}(2) \# 1-\operatorname{Ag}(1)-\operatorname{Ag}(2) \# 2$ & $143.553(13)$ \\
\hline $\operatorname{Ag}(1)-\operatorname{Ag}(2)$ & $2.8995(5)$ & $\operatorname{Se}(1) \# 2-\operatorname{Ag}(1)-\operatorname{Ag}(2) \# 2$ & $55.842(10)$ \\
\hline $\operatorname{Ag}(1)-\operatorname{Ag}(2) \# 2$ & $2.9866(4)$ & $\operatorname{Se}(2) \# 3-\operatorname{Ag}(1)-\operatorname{Ag}(2) \# 2$ & $102.273(12)$ \\
\hline $\operatorname{Ag}(1)-\operatorname{Ag}(2) \# 1$ & $3.0387(4)$ & $\operatorname{Ag}(2)-\operatorname{Ag}(1)-\operatorname{Ag}(2) \# 2$ & $106.864(10)$ \\
\hline $\operatorname{Se}(1)-C(11)$ & $1.930(3)$ & $\operatorname{Se}(1)-\operatorname{Ag}(1)-\operatorname{Ag}(2) \# 1$ & $142.448(13)$ \\
\hline $\operatorname{Se}(1)-\operatorname{Ag}(2)$ & $2.6885(4)$ & $\operatorname{Se}(2) \# 1-\operatorname{Ag}(1)-\operatorname{Ag}(2) \# 1$ & $55.710(10)$ \\
\hline $\operatorname{Se}(1)-\operatorname{Ag}(2) \# 2$ & $2.8596(4)$ & $\mathrm{Se}(1) \# 2-\mathrm{Ag}(1)-\mathrm{Ag}(2) \# 1$ & $97.920(12)$ \\
\hline$C(1)-C(6)$ & $1.393(4)$ & $\mathrm{Se}(2) \# 3-\mathrm{Ag}(1)-\mathrm{Ag}(2) \# 1$ & $54.755(10)$ \\
\hline$C(1)-C(2)$ & $1.397(4)$ & $\operatorname{Ag}(2)-\operatorname{Ag}(1)-\operatorname{Ag}(2) \# 1$ & $102.087(10)$ \\
\hline $\mathrm{C}(1)-\operatorname{Se}(2)$ & $1.924(3)$ & $\operatorname{Ag}(2) \# 2-\operatorname{Ag}(1)-\operatorname{Ag}(2) \# 1$ & $151.006(15)$ \\
\hline$C(2)-C(3)$ & $1.394(4)$ & $\mathrm{C}(11)-\operatorname{Se}(1)-\operatorname{Ag}(1)$ & $107.26(10)$ \\
\hline $\mathrm{C}(2)-\mathrm{H}(2)$ & 0.9500 & $\mathrm{C}(11)-\mathrm{Se}(1)-\operatorname{Ag}(2)$ & $103.16(10)$ \\
\hline$C(3)-C(4)$ & $1.390(5)$ & $\operatorname{Ag}(1)-\operatorname{Se}(1)-\operatorname{Ag}(2)$ & $65.274(12)$ \\
\hline $\mathrm{C}(3)-\mathrm{H}(3)$ & 0.9500 & $\mathrm{C}(11)-\mathrm{Se}(1)-\mathrm{Ag}(1) \# 4$ & $124.07(9)$ \\
\hline$C(4)-C(5)$ & $1.393(5)$ & $\operatorname{Ag}(1)-\operatorname{Se}(1)-\operatorname{Ag}(1) \# 4$ & $115.266(13)$ \\
\hline $\mathrm{C}(4)-\mathrm{H}(4)$ & 0.9500 & $\operatorname{Ag}(2)-\operatorname{Se}(1)-\operatorname{Ag}(1) \# 4$ & $66.816(11)$ \\
\hline$C(5)-C(6)$ & $1.393(4)$ & $\mathrm{C}(11)-\mathrm{Se}(1)-\operatorname{Ag}(2) \# 2$ & $127.05(9)$ \\
\hline $\mathrm{C}(5)-\mathrm{H}(5)$ & 0.9500 & $\operatorname{Ag}(1)-\operatorname{Se}(1)-\operatorname{Ag}(2) \# 2$ & $65.060(11)$ \\
\hline $\mathrm{C}(6)-\mathrm{H}(6)$ & 0.9500 & $\operatorname{Ag}(2)-\operatorname{Se}(1)-\operatorname{Ag}(2) \# 2$ & $116.855(13)$ \\
\hline $\operatorname{Ag}(2)-\operatorname{Se}(2)$ & $2.6984(4)$ & $\operatorname{Ag}(1) \# 4-\operatorname{Se}(1)-\operatorname{Ag}(2) \# 2$ & $103.594(14)$ \\
\hline $\operatorname{Ag}(2)-\operatorname{Se}(2) \# 1$ & $2.6993(4)$ & $\mathrm{C}(6)-\mathrm{C}(1)-\mathrm{C}(2)$ & $120.4(3)$ \\
\hline $\mathrm{C}(11)-\mathrm{C}(12)$ & $1.389(4)$ & $\mathrm{C}(6)-\mathrm{C}(1)-\mathrm{Se}(2)$ & $120.9(2)$ \\
\hline$C(11)-C(16)$ & $1.394(4)$ & $\mathrm{C}(2)-\mathrm{C}(1)-\mathrm{Se}(2)$ & $118.7(2)$ \\
\hline $\mathrm{C}(12)-\mathrm{C}(13)$ & $1.394(4)$ & $C(3)-C(2)-C(1)$ & $119.5(3)$ \\
\hline $\mathrm{C}(12)-\mathrm{H}(12)$ & 0.9500 & $\mathrm{C}(3)-\mathrm{C}(2)-\mathrm{H}(2)$ & 120.2 \\
\hline $\mathrm{C}(13)-\mathrm{C}(14)$ & $1.393(5)$ & $\mathrm{C}(1)-\mathrm{C}(2)-\mathrm{H}(2)$ & 120.2 \\
\hline $\mathrm{C}(13)-\mathrm{H}(13)$ & 0.9500 & $C(4)-C(3)-C(2)$ & $120.1(3)$ \\
\hline $\mathrm{C}(14)-\mathrm{C}(15)$ & $1.395(5)$ & $\mathrm{C}(4)-\mathrm{C}(3)-\mathrm{H}(3)$ & 119.9 \\
\hline $\mathrm{C}(14)-\mathrm{H}(14)$ & 0.9500 & $\mathrm{C}(2)-\mathrm{C}(3)-\mathrm{H}(3)$ & 119.9 \\
\hline$C(15)-C(16)$ & $1.391(4)$ & $C(3)-C(4)-C(5)$ & $120.2(3)$ \\
\hline $\mathrm{C}(15)-\mathrm{H}(15)$ & 0.9500 & $\mathrm{C}(3)-\mathrm{C}(4)-\mathrm{H}(4)$ & 119.9 \\
\hline \multirow[t]{2}{*}{$\mathrm{C}(16)-\mathrm{H}(16)$} & 0.9500 & $\mathrm{C}(5)-\mathrm{C}(4)-\mathrm{H}(4)$ & 119.9 \\
\hline & & $C(4)-C(5)-C(6)$ & $120.1(3)$ \\
\hline $\operatorname{Se}(1)-\operatorname{Ag}(1)-\operatorname{Se}(2) \# 1$ & $114.868(15)$ & $\mathrm{C}(4)-\mathrm{C}(5)-\mathrm{H}(5)$ & 120.0 \\
\hline $\operatorname{Se}(1)-\operatorname{Ag}(1)-\operatorname{Se}(1) \# 2$ & $116.018(11)$ & $\mathrm{C}(6)-\mathrm{C}(5)-\mathrm{H}(5)$ & 120.0 \\
\hline $\mathrm{Se}(2) \# 1-\mathrm{Ag}(1)-\mathrm{Se}(1) \# 2$ & $117.339(14)$ & $C(1)-C(6)-C(5)$ & 119.7(3) \\
\hline $\operatorname{Se}(1)-\operatorname{Ag}(1)-\operatorname{Se}(2) \# 3$ & $116.919(14)$ & $\mathrm{C}(1)-\mathrm{C}(6)-\mathrm{H}(6)$ & 120.2 \\
\hline $\mathrm{Se}(2) \# 1-\mathrm{Ag}(1)-\mathrm{Se}(2) \# 3$ & $110.387(11)$ & $\mathrm{C}(5)-\mathrm{C}(6)-\mathrm{H}(6)$ & 120.2 \\
\hline $\mathrm{Se}(1) \# 2-\mathrm{Ag}(1)-\mathrm{Se}(2) \# 3$ & $75.343(13)$ & $\operatorname{Se}(1)-\operatorname{Ag}(2)-\operatorname{Se}(2)$ & $120.631(15)$ \\
\hline $\operatorname{Se}(1)-\operatorname{Ag}(1)-\operatorname{Ag}(2)$ & $57.376(10)$ & $\operatorname{Se}(1)-\operatorname{Ag}(2)-\operatorname{Se}(2) \# 1$ & $114.908(15)$ \\
\hline $\operatorname{Se}(2) \# 1-\operatorname{Ag}(1)-\operatorname{Ag}(2)$ & $57.495(10)$ & $\operatorname{Se}(2)-\operatorname{Ag}(2)-\operatorname{Se}(2) \# 1$ & $114.127(11)$ \\
\hline
\end{tabular}




\begin{tabular}{|c|c|}
\hline $\operatorname{Se}(1)-\operatorname{Ag}(2)-\operatorname{Se}(1) \# 4$ & $111.963(11)$ \\
\hline $\operatorname{Se}(2)-\operatorname{Ag}(2)-\operatorname{Se}(1) \# 4$ & $75.180(13)$ \\
\hline $\operatorname{Se}(2) \# 1-\operatorname{Ag}(2)-\operatorname{Se}(1) \# 4$ & $113.692(14)$ \\
\hline $\operatorname{Se}(1)-\operatorname{Ag}(2)-\operatorname{Ag}(1)$ & $57.350(10)$ \\
\hline $\operatorname{Se}(2)-\operatorname{Ag}(2)-\operatorname{Ag}(1)$ & $148.134(14)$ \\
\hline $\operatorname{Se}(2) \# 1-\operatorname{Ag}(2)-\operatorname{Ag}(1)$ & $57.560(10)$ \\
\hline $\operatorname{Se}(1) \# 4-\operatorname{Ag}(2)-\operatorname{Ag}(1)$ & $136.609(14)$ \\
\hline $\operatorname{Se}(1)-\operatorname{Ag}(2)-\operatorname{Ag}(1) \# 4$ & $57.341(10)$ \\
\hline $\operatorname{Se}(2)-\operatorname{Ag}(2)-\operatorname{Ag}(1) \# 4$ & $100.352(13)$ \\
\hline $\operatorname{Se}(2) \# 1-\operatorname{Ag}(2)-\operatorname{Ag}(1) \# 4$ & $139.724(13)$ \\
\hline $\operatorname{Se}(1) \# 4-\operatorname{Ag}(2)-\operatorname{Ag}(1) \# 4$ & $54.691(9)$ \\
\hline $\operatorname{Ag}(1)-\operatorname{Ag}(2)-\operatorname{Ag}(1) \# 4$ & $102.182(10)$ \\
\hline $\operatorname{Se}(1)-\operatorname{Ag}(2)-\operatorname{Ag}(1) \# 5$ & $146.247(13)$ \\
\hline $\operatorname{Se}(2)-\operatorname{Ag}(2)-\operatorname{Ag}(1) \# 5$ & $55.797(10)$ \\
\hline $\operatorname{Se}(2) \# 1-\operatorname{Ag}(2)-\operatorname{Ag}(1) \# 5$ & $58.414(10)$ \\
\hline $\mathrm{Se}(1) \# 4-\mathrm{Ag}(2)-\mathrm{Ag}(1) \# 5$ & $99.693(12)$ \\
\hline $\operatorname{Ag}(1)-\operatorname{Ag}(2)-\operatorname{Ag}(1) \# 5$ & $106.448(10)$ \\
\hline $\operatorname{Ag}(1) \# 4-\operatorname{Ag}(2)-\operatorname{Ag}(1) \# 5$ & $151.007(15)$ \\
\hline$C(1)-\operatorname{Se}(2)-\operatorname{Ag}(2)$ & $121.12(9)$ \\
\hline $\mathrm{C}(1)-\mathrm{Se}(2)-\mathrm{Ag}(2) \# 5$ & 107.97(10) \\
\hline $\operatorname{Ag}(2)-\operatorname{Se}(2)-\operatorname{Ag}(2) \# 5$ & $117.663(14)$ \\
\hline $\mathrm{C}(1)-\mathrm{Se}(2)-\mathrm{Ag}(1) \# 5$ & $102.89(9)$ \\
\hline $\operatorname{Ag}(2)-\operatorname{Se}(2)-\operatorname{Ag}(1) \# 5$ & $68.492(11)$ \\
\hline $\operatorname{Ag}(2) \# 5-\operatorname{Se}(2)-\operatorname{Ag}(1) \# 5$ & $64.947(12)$ \\
\hline$C(1)-\operatorname{Se}(2)-\operatorname{Ag}(1) \# 6$ & $125.93(9)$ \\
\hline $\operatorname{Ag}(2)-\operatorname{Se}(2)-\operatorname{Ag}(1) \# 6$ & $105.762(14)$ \\
\hline $\operatorname{Ag}(2) \# 5-\operatorname{Se}(2)-\operatorname{Ag}(1) \# 6$ & $66.833(11)$ \\
\hline $\operatorname{Ag}(1) \# 5-\operatorname{Se}(2)-\operatorname{Ag}(1) \# 6$ & $119.139(13)$ \\
\hline$C(12)-C(11)-C(16)$ & $120.5(3)$ \\
\hline $\mathrm{C}(12)-\mathrm{C}(11)-\mathrm{Se}(1)$ & $118.6(2)$ \\
\hline$C(16)-C(11)-S e(1)$ & $120.9(2)$ \\
\hline$C(11)-C(12)-C(13)$ & $119.6(3)$ \\
\hline $\mathrm{C}(11)-\mathrm{C}(12)-\mathrm{H}(12)$ & 120.2 \\
\hline $\mathrm{C}(13)-\mathrm{C}(12)-\mathrm{H}(12)$ & 120.2 \\
\hline$C(14)-C(13)-C(12)$ & $120.2(3)$ \\
\hline $\mathrm{C}(14)-\mathrm{C}(13)-\mathrm{H}(13)$ & 119.9 \\
\hline $\mathrm{C}(12)-\mathrm{C}(13)-\mathrm{H}(13)$ & 119.9 \\
\hline $\mathrm{C}(13)-\mathrm{C}(14)-\mathrm{C}(15)$ & $119.8(3)$ \\
\hline $\mathrm{C}(13)-\mathrm{C}(14)-\mathrm{H}(14)$ & 120.1 \\
\hline $\mathrm{C}(15)-\mathrm{C}(14)-\mathrm{H}(14)$ & 120.1 \\
\hline$C(16)-C(15)-C(14)$ & $120.1(3)$ \\
\hline $\mathrm{C}(16)-\mathrm{C}(15)-\mathrm{H}(15)$ & 119.9 \\
\hline $\mathrm{C}(14)-\mathrm{C}(15)-\mathrm{H}(15)$ & 119.9 \\
\hline$C(15)-C(16)-C(11)$ & $119.7(3)$ \\
\hline $\mathrm{C}(15)-\mathrm{C}(16)-\mathrm{H}(16)$ & 120.2 \\
\hline $\mathrm{C}(11)-\mathrm{C}(16)-\mathrm{H}(16)$ & 120.2 \\
\hline
\end{tabular}

Symmetry transformations used to generate equivalent atoms: $\# 1-\mathrm{x}+1, \mathrm{y}+1 / 2,-\mathrm{z}+1 / 2$ \#2 -x,y+1/2,-z+1/2 \#3 x,y+1,z \#4 -x,y-1/2,-z+1/2 \#5-x+1,y-1/2,-z+1/2 \#6 x,y-1,z 
Table S3. Anisotropic displacement parameters $\left(\AA^{2} \mathrm{x} 10^{3}\right)$ for $\mathrm{AgSePh}$. The anisotropic displacement factor exponent takes the form: $-2 p^{2}\left[h^{2} a^{* 2} U^{11}+\ldots+2 h k a^{*} b^{*} U^{12}\right]$

\begin{tabular}{lcccccc}
\hline & $\mathrm{U}^{11}$ & $\mathrm{U}^{22}$ & $\mathrm{U}^{33}$ & $\mathrm{U}^{23}$ & $\mathrm{U}^{13}$ & $\mathrm{U}^{12}$ \\
\hline $\mathrm{Ag}(1)$ & $17(1)$ & $12(1)$ & $26(1)$ & $1(1)$ & $4(1)$ & $1(1)$ \\
$\mathrm{Se}(1)$ & $13(1)$ & $11(1)$ & $15(1)$ & $0(1)$ & $0(1)$ & $0(1)$ \\
$\mathrm{C}(1)$ & $13(1)$ & $13(1)$ & $16(1)$ & $2(1)$ & $0(1)$ & $-2(1)$ \\
$\mathrm{C}(2)$ & $16(1)$ & $19(2)$ & $18(1)$ & $2(1)$ & $3(1)$ & $2(1)$ \\
$\mathrm{C}(3)$ & $22(2)$ & $21(2)$ & $17(1)$ & $1(1)$ & $5(1)$ & $1(1)$ \\
$\mathrm{C}(4)$ & $20(2)$ & $20(2)$ & $17(2)$ & $1(1)$ & $-2(1)$ & $-2(1)$ \\
$\mathrm{C}(5)$ & $17(1)$ & $20(2)$ & $21(2)$ & $1(1)$ & $-2(1)$ & $1(1)$ \\
$\mathrm{C}(6)$ & $15(1)$ & $16(1)$ & $19(1)$ & $0(1)$ & $0(1)$ & $2(1)$ \\
$\mathrm{Ag}(2)$ & $18(1)$ & $11(1)$ & $26(1)$ & $1(1)$ & $3(1)$ & $1(1)$ \\
$\mathrm{Se}(2)$ & $13(1)$ & $11(1)$ & $15(1)$ & $0(1)$ & $0(1)$ & $0(1)$ \\
$\mathrm{C}(11)$ & $14(1)$ & $13(1)$ & $16(1)$ & $0(1)$ & $-2(1)$ & $0(1)$ \\
$\mathrm{C}(12)$ & $17(1)$ & $17(1)$ & $18(1)$ & $0(1)$ & $2(1)$ & $-2(1)$ \\
$\mathrm{C}(13)$ & $22(2)$ & $20(2)$ & $19(2)$ & $2(1)$ & $4(1)$ & $0(1)$ \\
$\mathrm{C}(14)$ & $22(2)$ & $19(2)$ & $17(2)$ & $0(1)$ & $-2(1)$ & $2(1)$ \\
$\mathrm{C}(15)$ & $17(1)$ & $20(2)$ & $20(2)$ & $-1(1)$ & $-1(1)$ & $-1(1)$ \\
$\mathrm{C}(16)$ & $16(1)$ & $16(1)$ & $19(1)$ & $2(1)$ & $1(1)$ & $-1(1)$ \\
\hline
\end{tabular}


Table S4. Hydrogen coordinates $\left(x 10^{4}\right)$ and isotropic displacement parameters $\left(\AA^{2}\right.$ x $\left.10^{3}\right)$ for $\mathrm{AgSePh}$.

\begin{tabular}{lrrrl}
\hline & $x$ & $y$ & $z$ & $U(e q)$ \\
& & & & \\
$H(2)$ & 3112 & 5652 & 3822 & 21 \\
$H(3)$ & 4579 & 5630 & 4602 & 24 \\
$H(4)$ & 8164 & 4301 & 4823 & 23 \\
$H(5)$ & 10337 & 3040 & 4266 & 23 \\
$H(6)$ & 8917 & 3102 & 3483 & 20 \\
$H(12)$ & -1981 & 8062 & 3843 & 21 \\
$H(13)$ & -575 & 8172 & 4626 & 24 \\
$H(14)$ & 3023 & 9493 & 4849 & 24 \\
$H(15)$ & 5263 & 10640 & 4286 & 23 \\
$H(16)$ & 3879 & 10503 & 3503 & 20 \\
\hline
\end{tabular}


Table S5. Atomic coordinates (x 104) and equivalent isotropic displacement parameters $\left(\AA^{2} \mathrm{x}\right.$ $10^{3}$ ) for $\mathrm{AgSePhMe}$. $\mathrm{U}(\mathrm{eq})$ is defined as one third of the trace of the orthogonalized Uij tensor.

\begin{tabular}{lcccc}
\hline & $\mathrm{x}$ & $\mathrm{y}$ & $\mathrm{z}$ & $\mathrm{U}(\mathrm{eq})$ \\
\hline $\mathrm{Ag}(1)$ & & & \\
$\mathrm{Se}(1)$ & $4942(1)$ & $3035(1)$ & $4816(1)$ & $20(1)$ \\
$\mathrm{C}(1)$ & $5790(1)$ & $5018(1)$ & $2180(1)$ & $14(1)$ \\
$\mathrm{C}(2)$ & $6847(2)$ & $5053(3)$ & $3851(4)$ & $15(1)$ \\
$\mathrm{C}(3)$ & $7446(2)$ & $5775(3)$ & $2772(4)$ & $18(1)$ \\
$\mathrm{C}(4)$ & $8219(2)$ & $5694(4)$ & $3923(5)$ & $22(1)$ \\
$\mathrm{C}(5)$ & $8406(2)$ & $4897(3)$ & $6145(5)$ & $20(1)$ \\
$\mathrm{C}(6)$ & $7797(2)$ & $4203(3)$ & $7213(4)$ & $19(1)$ \\
$\mathrm{C}(7)$ & $7022(1)$ & $4285(3)$ & $6091(4)$ & $17(1)$ \\
& $9242(2)$ & $4781(5)$ & $7388(7)$ & $31(1)$ \\
\hline
\end{tabular}


Table S6. Bond lengths $[\AA]$ and angles $\left[{ }^{\circ}\right]$ for $\mathrm{AgSePh}$.

\begin{tabular}{|c|c|c|c|}
\hline $\operatorname{Ag}(1)-\operatorname{Se}(1)$ & $2.6909(3)$ & $\mathrm{C}(1)-\operatorname{Se}(1)-\operatorname{Ag}(1)$ & $106.41(8)$ \\
\hline $\operatorname{Ag}(1)-\operatorname{Se}(1) \# 1$ & $2.6961(3)$ & $\mathrm{C}(1)-\mathrm{Se}(1)-\mathrm{Ag}(1) \# 5$ & $123.11(7)$ \\
\hline $\operatorname{Ag}(1)-\operatorname{Se}(1) \# 2$ & $2.7009(3)$ & $\operatorname{Ag}(1)-\operatorname{Se}(1)-\operatorname{Ag}(1) \# 5$ & $114.598(10)$ \\
\hline $\operatorname{Ag}(1)-\operatorname{Ag}(1) \# 2$ & $2.8682(4)$ & $\mathrm{C}(1)-\operatorname{Se}(1)-\operatorname{Ag}(1) \# 2$ & $100.08(8)$ \\
\hline $\operatorname{Ag}(1)-\operatorname{Se}(1) \# 3$ & $2.8797(3)$ & $\operatorname{Ag}(1)-\operatorname{Se}(1)-\operatorname{Ag}(1) \# 2$ & $64.274(10)$ \\
\hline $\operatorname{Ag}(1)-\operatorname{Ag}(1) \# 3$ & $2.98675(18)$ & $\operatorname{Ag}(1) \# 5-\operatorname{Se}(1)-\operatorname{Ag}(1) \# 2$ & $67.202(7)$ \\
\hline $\operatorname{Ag}(1)-\operatorname{Ag}(1) \# 4$ & $2.98676(18)$ & $\mathrm{C}(1)-\mathrm{Se}(1)-\operatorname{Ag}(1) \# 4$ & $128.21(7)$ \\
\hline $\operatorname{Se}(1)-C(1)$ & $1.925(2)$ & $\operatorname{Ag}(1)-\operatorname{Se}(1)-\operatorname{Ag}(1) \# 4$ & $64.742(7)$ \\
\hline$C(1)-C(2)$ & $1.391(4)$ & $\operatorname{Ag}(1) \# 5-\operatorname{Se}(1)-\operatorname{Ag}(1) \# 4$ & $104.786(10)$ \\
\hline$C(1)-C(6)$ & $1.396(3)$ & $\operatorname{Ag}(1) \# 2-\operatorname{Se}(1)-\operatorname{Ag}(1) \# 4$ & $116.897(10)$ \\
\hline$C(2)-C(3)$ & $1.396(3)$ & $\mathrm{C}(2)-\mathrm{C}(1)-\mathrm{C}(6)$ & $119.8(2)$ \\
\hline $\mathrm{C}(2)-\mathrm{H}(2)$ & 0.9500 & $\mathrm{C}(2)-\mathrm{C}(1)-\mathrm{Se}(1)$ & 119.63(19) \\
\hline$C(3)-C(4)$ & $1.397(4)$ & $\mathrm{C}(6)-\mathrm{C}(1)-\mathrm{Se}(1)$ & $120.48(19)$ \\
\hline $\mathrm{C}(3)-\mathrm{H}(3)$ & 0.9500 & $\mathrm{C}(1)-\mathrm{C}(2)-\mathrm{C}(3)$ & $119.6(2)$ \\
\hline $\mathrm{C}(4)-\mathrm{C}(5)$ & $1.395(4)$ & $\mathrm{C}(1)-\mathrm{C}(2)-\mathrm{H}(2)$ & 120.2 \\
\hline$C(4)-C(7)$ & $1.510(4)$ & $\mathrm{C}(3)-\mathrm{C}(2)-\mathrm{H}(2)$ & 120.2 \\
\hline$C(5)-C(6)$ & $1.393(3)$ & $\mathrm{C}(2)-\mathrm{C}(3)-\mathrm{C}(4)$ & $121.3(3)$ \\
\hline $\mathrm{C}(5)-\mathrm{H}(5)$ & 0.9500 & $\mathrm{C}(2)-\mathrm{C}(3)-\mathrm{H}(3)$ & 119.4 \\
\hline $\mathrm{C}(6)-\mathrm{H}(6)$ & 0.9500 & $\mathrm{C}(4)-\mathrm{C}(3)-\mathrm{H}(3)$ & 119.4 \\
\hline $\mathrm{C}(7)-\mathrm{H}(7 \mathrm{~A})$ & 0.9800 & $\mathrm{C}(5)-\mathrm{C}(4)-\mathrm{C}(3)$ & $118.3(2)$ \\
\hline $\mathrm{C}(7)-\mathrm{H}(7 \mathrm{~B})$ & 0.9800 & $C(5)-C(4)-C(7)$ & $120.1(3)$ \\
\hline $\mathrm{C}(7)-\mathrm{H}(7 \mathrm{C})$ & 0.9800 & $\mathrm{C}(3)-\mathrm{C}(4)-\mathrm{C}(7)$ & $121.6(3)$ \\
\hline & & $\mathrm{C}(6)-\mathrm{C}(5)-\mathrm{C}(4)$ & $121.1(2)$ \\
\hline $\operatorname{Se}(1)-\operatorname{Ag}(1)-\operatorname{Se}(1) \# 1$ & $117.032(9)$ & $\mathrm{C}(6)-\mathrm{C}(5)-\mathrm{H}(5)$ & 119.5 \\
\hline $\operatorname{Se}(1)-\operatorname{Ag}(1)-\operatorname{Se}(1) \# 2$ & $115.726(10)$ & $\mathrm{C}(4)-\mathrm{C}(5)-\mathrm{H}(5)$ & 119.5 \\
\hline $\mathrm{Se}(1) \# 1-\mathrm{Ag}(1)-\mathrm{Se}(1) \# 2$ & $117.676(13)$ & $\mathrm{C}(5)-\mathrm{C}(6)-\mathrm{C}(1)$ & $119.9(2)$ \\
\hline $\operatorname{Se}(1)-\operatorname{Ag}(1)-\operatorname{Ag}(1) \# 2$ & $58.034(9)$ & $\mathrm{C}(5)-\mathrm{C}(6)-\mathrm{H}(6)$ & 120.1 \\
\hline $\operatorname{Se}(1) \# 1-\operatorname{Ag}(1)-\operatorname{Ag}(1) \# 2$ & $149.744(13)$ & $\mathrm{C}(1)-\mathrm{C}(6)-\mathrm{H}(6)$ & 120.1 \\
\hline $\operatorname{Se}(1) \# 2-\operatorname{Ag}(1)-\operatorname{Ag}(1) \# 2$ & $57.692(8)$ & $\mathrm{C}(4)-\mathrm{C}(7)-\mathrm{H}(7 \mathrm{~A})$ & 109.5 \\
\hline $\operatorname{Se}(1)-\operatorname{Ag}(1)-\operatorname{Se}(1) \# 3$ & $113.464(12)$ & $\mathrm{C}(4)-\mathrm{C}(7)-\mathrm{H}(7 \mathrm{~B})$ & 109.5 \\
\hline $\mathrm{Se}(1) \# 1-\mathrm{Ag}(1)-\mathrm{Se}(1) \# 3$ & 75.213(10) & H(7A)-C(7)-H(7B) & 109.5 \\
\hline $\operatorname{Se}(1) \# 2-\operatorname{Ag}(1)-\operatorname{Se}(1) \# 3$ & $110.770(8)$ & $\mathrm{C}(4)-\mathrm{C}(7)-\mathrm{H}(7 \mathrm{C})$ & 109.5 \\
\hline $\operatorname{Ag}(1) \# 2-\operatorname{Ag}(1)-\operatorname{Se}(1) \# 3$ & $135.031(12)$ & $\mathrm{H}(7 \mathrm{~A})-\mathrm{C}(7)-\mathrm{H}(7 \mathrm{C})$ & 109.5 \\
\hline $\operatorname{Se}(1)-\operatorname{Ag}(1)-\operatorname{Ag}(1) \# 3$ & $140.200(8)$ & $\mathrm{H}(7 \mathrm{~B})-\mathrm{C}(7)-\mathrm{H}(7 \mathrm{C})$ & 109.5 \\
\hline $\operatorname{Se}(1) \# 1-\operatorname{Ag}(1)-\operatorname{Ag}(1) \# 3$ & $97.409(11)$ & & \\
\hline $\operatorname{Se}(1) \# 2-\operatorname{Ag}(1)-\operatorname{Ag}(1) \# 3$ & $56.320(7)$ & \multirow{9}{*}{\multicolumn{2}{|c|}{ 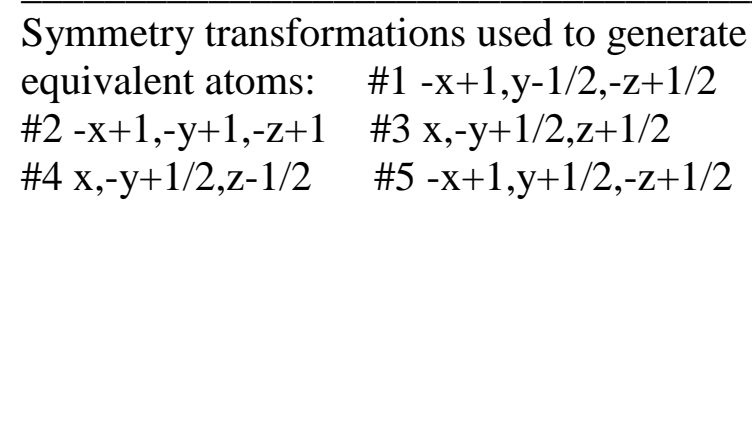 }} \\
\hline $\operatorname{Ag}(1) \# 2-\operatorname{Ag}(1)-\operatorname{Ag}(1) \# 3$ & $101.453(10)$ & & \\
\hline $\operatorname{Se}(1) \# 3-\operatorname{Ag}(1)-\operatorname{Ag}(1) \# 3$ & $54.568(8)$ & & \\
\hline $\operatorname{Se}(1)-\operatorname{Ag}(1)-\operatorname{Ag}(1) \# 4$ & $60.690(7)$ & & \\
\hline $\operatorname{Se}(1) \# 1-\operatorname{Ag}(1)-\operatorname{Ag}(1) \# 4$ & $56.476(8)$ & & \\
\hline $\operatorname{Se}(1) \# 2-\operatorname{Ag}(1)-\operatorname{Ag}(1) \# 4$ & $145.817(9)$ & & \\
\hline $\operatorname{Ag}(1) \# 2-\operatorname{Ag}(1)-\operatorname{Ag}(1) \# 4$ & $108.647(11)$ & & \\
\hline $\operatorname{Se}(1) \# 3-\operatorname{Ag}(1)-\operatorname{Ag}(1) \# 4$ & $100.297(10)$ & & \\
\hline $\operatorname{Ag}(1) \# 3-\operatorname{Ag}(1)-\operatorname{Ag}(1) \# 4$ & $149.825(15)$ & & \\
\hline
\end{tabular}


Table S7. Anisotropic displacement parameters $\left(\AA^{2} \times 10^{3}\right)$ for AgSePhMe. The anisotropic displacement factor exponent takes the form: $-2 p^{2}\left[h^{2} a^{* 2} U^{11}+\ldots+2 h k a^{*} b^{*} U^{12}\right]$

\begin{tabular}{lcccccc}
\hline & $\mathrm{U}^{11}$ & $\mathrm{U}^{22}$ & $\mathrm{U}^{33}$ & $\mathrm{U}^{23}$ & $\mathrm{U}^{13}$ & $\mathrm{U}$ \\
\hline $\mathrm{Ag}(1)$ & $26(1)$ & $13(1)$ & $20(1)$ & $-2(1)$ & $4(1)$ & $-2(1)$ \\
$\mathrm{Se}(1)$ & $16(1)$ & $12(1)$ & $15(1)$ & $0(1)$ & $-1(1)$ & $0(1)$ \\
$\mathrm{C}(1)$ & $15(1)$ & $14(1)$ & $14(1)$ & $0(1)$ & $-1(1)$ & $1(1)$ \\
$\mathrm{C}(2)$ & $22(1)$ & $16(1)$ & $17(1)$ & $1(1)$ & $3(1)$ & $0(1)$ \\
$\mathrm{C}(3)$ & $18(1)$ & $22(1)$ & $25(1)$ & $0(1)$ & $4(1)$ & $-1(1)$ \\
$\mathrm{C}(4)$ & $18(1)$ & $19(1)$ & $23(1)$ & $-2(1)$ & $0(1)$ & $1(1)$ \\
$\mathrm{C}(5)$ & $21(1)$ & $18(1)$ & $17(1)$ & $0(1)$ & $-2(1)$ & $2(1)$ \\
$\mathrm{C}(6)$ & $18(1)$ & $16(1)$ & $16(1)$ & $1(1)$ & $2(1)$ & $-1(1)$ \\
$\mathrm{C}(7)$ & $17(1)$ & $37(2)$ & $35(2)$ & $0(1)$ & $-5(1)$ & $1(1)$ \\
\hline
\end{tabular}


Table S8. Hydrogen coordinates $\left(x 10^{4}\right)$ and isotropic displacement parameters $\left(\AA^{2} \times 10^{3}\right)$ for $\mathrm{AgSePh}$.

\begin{tabular}{lcccc}
\hline & $\mathrm{x}$ & $\mathrm{y}$ & $\mathrm{z}$ & $\mathrm{U}(\mathrm{eq})$ \\
& & & & \\
$\mathrm{H}(2)$ & 7329 & 6319 & 1259 & 22 \\
$\mathrm{H}(3)$ & 8626 & 6190 & 3181 & 26 \\
$\mathrm{H}(5)$ & 7913 & 3666 & 8730 & 23 \\
$\mathrm{H}(6)$ & 6613 & 3818 & 6850 & 21 \\
$\mathrm{H}(7 \mathrm{~A})$ & 9464 & 3583 & 7067 & 46 \\
$\mathrm{H}(7 \mathrm{~B})$ & 9253 & 4918 & 9083 & 46 \\
$\mathrm{H}(7 \mathrm{C})$ & 9553 & 5764 & 6821 & 46 \\
\hline
\end{tabular}

